\title{
Synthesis of New Quinolone Derivatives Linked to Benzothiazole or Benzoxazole Moieties as Anticancer and Anti-Oxidant Agents
}

\section{Mohamed A Abdelgawad ${ }^{1,2 *}$, Phoebe F Lamie ${ }^{1 *}$ and Osama M Ahmed ${ }^{3}$}

${ }^{1}$ Pharmaceutical Organic Chemistry Department, Faculty of Pharmacy, Beni-Suef University, Beni Suef, Egypt ${ }^{2}$ Pharmaceutical Chemistry Department, College of Pharmacy, Al Jouf University, Sakaka, Al Jouf, Saudi Arabia ${ }^{3}$ Zoology Department, Faculty of Science, Beni-Suef University, Beni Suef, Egypt

\begin{abstract}
A new series of substituted quinolones linked to benzothiazole and/or benzoxazole moieties $5 \mathrm{a}-\mathrm{I}$ was synthesized. 6-Benzoxazol-2-yl/benzothiazol-2-yl-4-oxo-1,4-dihydro-quinoline-3-carboxylic acid ethyl esters 3a\&b were reacted with hydrazine to give the hydrazide derivatives $4 a \& b$ and finally, $4 a \& b$ were reacted with different aromatic aldehydes giving the target compounds 5a-I. The benzylidene derivatives 5a-I were screened for their cytotoxic activities against breast carcinoma cell lines (MCF-7) and anti-oxidant properties. All the tested compounds $5 a-I$ showed from high to moderate activity as anticancer and anti-oxidant agents. Compounds $5 \mathrm{~h}$ and $5 \mathrm{l}$ showed the highest cytotoxicr activity against MCF-7 (IC 50.058 and $0.052 \mathrm{uM}$, respectively) than 4-(benzothiazol-2-yl) aniline the reference drug $\left(\mathrm{IC}_{50}: 0.065 \mathrm{uM}\right)$. Moreover, compounds $5 \mathrm{e}, 5 \mathrm{~g}$ and $5 \mathrm{~h}$ showed the highest anti-oxidant activity. The structure of the compounds 5 a-I was confirmed using IR, ${ }^{1} \mathrm{H}$ NMR, mass spectroscopy and elemental analysis.
\end{abstract}

Keywords: Quinolones; Benzothiazoles; Benzoxazoles; Anti-oxidant activity; Anticancer effect

\section{Introduction}

The cytotoxic activity of quinolone derivatives has become the source of new anticancer agents, which might also help addressing side-toxicity and resistance [1]. Moreover, the quinolone ring is considered an important structural unit in many anti-oxidant agents [2]. New synthesized 4-arylchalcogenyl-7-chloroquinolines were screened in vitro for antioxidant activity by previous publication which demonstrated that compound presented a potent antioxidant effect [3].

Quinolones were used especially as radicals scavenger like quercetol (A) or coumestrol (B) and the copper or iron chelating molecules such as clioquinol (C) [4,5]. Moreover, quinolone containing hydroxyl group compounds exhibited antiradical activity against DPPH radical and anion superoxide tests activity [6].

On the other hand, benzothiazoles showed potent scavenging activitiesagainstDPPHradicaland2,2'-azino-bis(3-ethylbenzthiazoline6-sulphonic acid) (ABTS) (D) radicals had reducing power, and strong inhibitory capacity on lipid peroxidation. Also, benzothiazoles or benzoxazoles containing compounds were found to be cytotoxic against CNS cancer cell line SNB-75 [7]. Moreover, 4-(benzothiol-2yl) aniline (Eb) showed a promising cytotoxic activity against breast, overian, lung and renal cell lines [8-10]. Activity was partially retained in benzoxazole analogue Ea [8] (Figure 1). Compounds of thiol and aminothiol derived from benzothiazoles showed an promising antioxidant property [11].

Benzothiazoles and benzoxazoles containing compounds were showed anticancer activity against various cell lines [12,13]. New synthesized compounds containing benzothiazoles or benzoxazoles linked to quinolone showed anticancer and antimicrobial activities $[14,15]$.

According to the aforementioned facts and as a continuation of our previous studies in the field of anticancer screening and anti-oxidant evaluation, [16-19] we attempt to design novel quinolone derivatives through:

- Substitution at quinolone nucleus with benzothiazole and benzoxazole rings (which have antioxidant or anticancer activity) at 6 position of quinolone.

- Maintain the main structure which responsible for receptor coupling of quinolone.

- Substitution of carboxyl group at 3-postion by substituted phenylhydrazone to increase its lipophilicity.

- Over all incorporation of benzoxazole or benzothiazole and quinolone in one scaffold structure.

All the synthesized compounds were evaluated for their anticancer activity against human breast adenocarcinoma cell line (MCF-7) and antioxidant activity.

\section{Materials and Methods}

\section{Chemistry}

Melting points were determined on a Graffin apparatus and were uncorrected. Element analyses $(\mathrm{C}, \mathrm{H}$, and $\mathrm{N})$ were carried out on Perkin-Elmer 2400 analyzer (Perkin-Elmer, Norwalk, CT, USA) at the Micro analytical unit of Cairo University, Egypt. All compounds were within $\pm 0.4 \%$ of the theoretical values. IR spectra were determined as $\mathrm{KBr}$ discs on Shimadzu IR 435 Spectrophotometer and values were represented in $\mathrm{cm}^{-1} .{ }^{1} \mathrm{H}$ NMR spectra were carried out on a Bruker 400

*Corresponding authors: Mohamed A Abdelgawad, Pharmaceutical Chemistry Department, College of Pharmacy, Al Jouf University, Sakaka, Al Jouf-2014, Saudi Arabia, E-mail: mohamed.abdelgawad@pharm.bsu.edu.eg

Phoebe F Lamie, Pharmaceutical Organic Chemistry Department, Faculty of Pharmacy, Beni-Suef University, Beni-Suef, Egypt, Tel: 002012233214514; E-mail feby.farag@yahoo.com

Received October 12, 2016; Accepted October 24, 2016; Published October 27 2016

Citation: Abdelgawad MA, Lamie PF, Ahmed OM (2016) Synthesis of New Quinolone Derivatives Linked to Benzothiazole or Benzoxazole Moieties as Anticancer and Anti-Oxidant Agents. Med Chem (Los Angeles) 6: 652-657. doi:10.4172/2161-0444.1000410

Copyright: (c) 2016 Abdelgawad MA, et al. This is an open-access article distributed under the terms of the Creative Commons Attribution License, which permits unrestricted use, distribution, and reproduction in any medium, provided the original author and source are credited. 

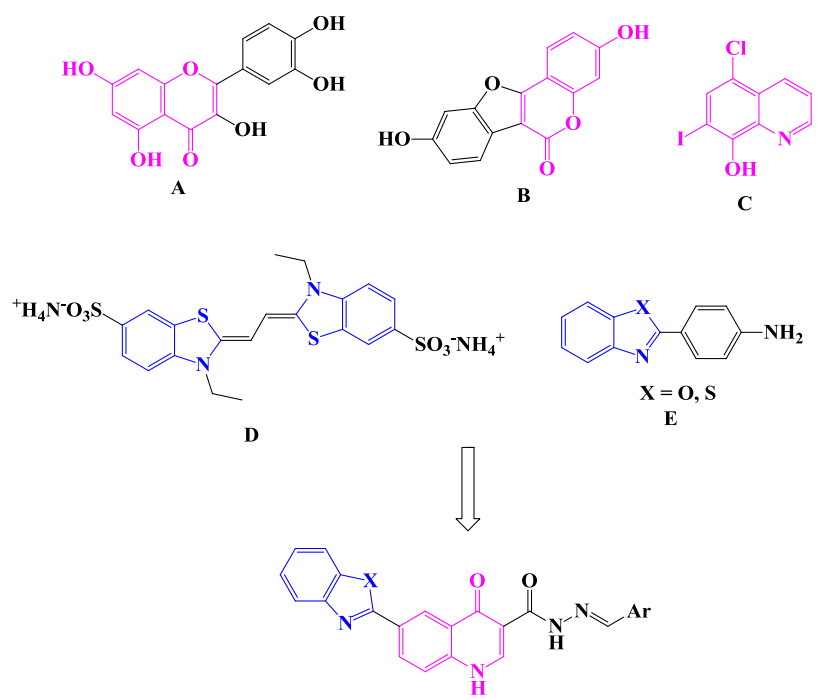

Target compounds 5a-1

Figure 1: Chemical structures of previously synthesized anticancer and antioxidant agents containing benzoxazole, benzothiazole and quinolone moieties (A-E) and the design of the target compounds 5a-1.

MHz NMR Spectrophotometer in Beni Suef University, Beni Suef, Egypt, using (Bruker, Munich, Germany) in DMSO- $d_{6}$ as a solvent, TMS as internal standard and chemical shifts were recorded in ppm on $\delta$ scale. Mass spectra were run on Hewlett Packard 5988 Spectrometer, Micro analytical center, Cairo University, Egypt. Progress of the reactions was monitored by TLC using TLC sheets percolated with UV fluorescent silica gel MERCK $60 \mathrm{~F} 254$ that were visualized by UV lamp.

General procedure for the synthesis of 6-(benzo[d] oxazole or thiazol-2-yl)-4-oxo-1,4-dihydroquinoline-3-carbohydrazide $4 a \& b$

To a suspension of compounds $3 \mathrm{a} \& \mathrm{~b}(0.01 \mathrm{~mol})$ in absolute ethanol (30 mL), hydrazine hydrate $99 \%$ (5 g, $0.1 \mathrm{~mol}$ ) was added. The mixture was heated under reflux for $20 \mathrm{~h}$. The precipitated solid was filtered, dried and crystallized from DMF/ethanol.

6-(Benzo[d]oxazole-2-yl)-4-oxo-1,4-dihydroquinoline-3carbohydrazide (4a): Yield: 53\%; yellow crystals ;mp: $390-392^{\circ} \mathrm{C}$; IR $\left(\mathrm{cm}^{-1}\right)$ : 3465-3192 (2NH and $\left.\mathrm{NH}_{2}\right), 1661,1613(2 \mathrm{C}=\mathrm{O}) ;{ }^{1} \mathrm{H}$ NMR (DMSO- $\left.d_{6}\right) \delta$ ppm $4.65\left(\mathrm{~s}, 2 \mathrm{H}, \mathrm{NH}_{2}, \mathrm{D}_{2} \mathrm{O}\right.$ exchangeable), $7.47(\mathrm{~m}, 2 \mathrm{H}$, Ar-H), 7.86 (m, 2H, Ar-H), $7.92(\mathrm{~d}, 1 \mathrm{H}, J=9.2 \mathrm{~Hz}, \mathrm{Ar}-\mathrm{H}), 8.53$ (d, $1 \mathrm{H}$, $J=7.6 \mathrm{~Hz}, \mathrm{Ar}-\mathrm{H}), 8.82(\mathrm{~s}, 1 \mathrm{H}, \mathrm{Ar}-\mathrm{H}), 9.04(\mathrm{~s}, 1 \mathrm{H}, \mathrm{Ar}-\mathrm{H}), 10.63(\mathrm{~s}, 1 \mathrm{H}$, CONH, $\mathrm{D}_{2} \mathrm{O}$ exchangeable), $13.06\left(\mathrm{~s}, 1 \mathrm{H}, \mathrm{NH}, \mathrm{D}_{2} \mathrm{O}\right.$ exchangeable); $\mathrm{MS}$ $\mathrm{m} / z: 320\left[(\mathrm{M})^{+}, 7.98 \%\right], 288\left[\left(\mathrm{C}_{17} \mathrm{H}_{8} \mathrm{~N}_{2} \mathrm{O}_{3}\right)^{+}, 100 \%\right]$. Anal. Calcd. for $\mathrm{C}_{17} \mathrm{H}_{12} \mathrm{~N}_{4} \mathrm{O}_{3}: \mathrm{C}, 63.75 ; \mathrm{H}, 3.78 ; \mathrm{N}, 17.49$. Found: C, 63.70; H, 3.50; N, 17.20 .

6-(Benzo[d] thiazol-2-yl)-4-oxo-1,4-dihydroquinoline-3carbohydrazide (4b): Yield: 53\%; yellow crystals; mp: 396-398 ${ }^{\circ} \mathrm{C}$; IR $\left(\mathrm{cm}^{-1}\right)$ : 3422-3176 (2NH and $\left.\mathrm{NH}_{2}\right), 1661,1624(2 \mathrm{C}=\mathrm{O}) ;{ }^{1} \mathrm{H}$ NMR (DMSO- $\left.d_{6}\right) \delta \mathrm{ppm} 4.51$ (s, 2H, $\mathrm{NH}_{2}, \mathrm{D}_{2} \mathrm{O}$ exchangeable), $7.49(\mathrm{~m}, 1 \mathrm{H}$, Ar-H), $7.57(\mathrm{~m}, 1 \mathrm{H}, \mathrm{Ar}-\mathrm{H}), 7.84(\mathrm{~d}, 1 \mathrm{H}, J=8.4 \mathrm{~Hz}, \mathrm{Ar}-\mathrm{H}), 8.11(\mathrm{~d}, 1 \mathrm{H}$, $J=7.2 \mathrm{~Hz}, \mathrm{Ar}-\mathrm{H}), 8.18(\mathrm{~d}, 1 \mathrm{H}, J=7.2 \mathrm{~Hz}, \mathrm{Ar}-\mathrm{H}), 8.40(\mathrm{~d}, 1 \mathrm{H}, J=8 \mathrm{~Hz}$, Ar-H), 8.81 (s, 1H, Ar-H), 8.89 (s, 1H, Ar-H), 10.84 (s, 1H, CONH, $\mathrm{D}_{2} \mathrm{O}$ exchangeable), 13.29 (s, $1 \mathrm{H}, \mathrm{NH}, \mathrm{D}_{2} \mathrm{O}$ exchangeable); $\mathrm{MS} m / z: 336$ $\left[(\mathrm{M})^{++}, 20.70 \%\right], 57\left[\left(\mathrm{C}_{4} \mathrm{H}_{9}\right)^{+}, 100 \%\right]$. Anal. Calcd. For $\mathrm{C}_{17} \mathrm{H}_{12} \mathrm{~N}_{4} \mathrm{O}_{2} \mathrm{~S}: \mathrm{C}$, $60.70 ; \mathrm{H}, 3.60 ; \mathrm{N}, 16.66$. Found: C, 60.60; H, 3.40; N, 16.50 .
General procedure for the synthesis of (ZE)-6-(benzo[d] oxazol or thiazol-2-yl)-N'-substituted benzylidene-4-oxo-1,4dihydroquinoline-3-carbohydrazide 5a-1

A mixture of $4 \mathbf{a} \& \mathbf{b}(0.01 \mathrm{~mol})$ and the appropriate aromatic aldehyde $(0.01 \mathrm{~mol})$ in absolute ethanol, catalytic amount of glacial acetic acid was added $(0.5 \mathrm{~mL})$. The reaction mixture was heated under reflux for 8-10 h (monitored by TLC). The separated solid was filtered, dried and crystallized from DMF.

(ZE)6-(Benzo[d] oxazol-2-yl)-N'-benzylidene-4-oxo-1,4dihydroquinoline-3-carbohydrazide (5a): Yield: 53\%; yellow solid; mp: $349-351^{\circ} \mathrm{C}$; IR $\left(\mathrm{cm}^{-1}\right): 3439,3141(2 \mathrm{NH}), 3089$ (CH aromatic), 1697, $1615(2 \mathrm{C}=\mathrm{O}) ;{ }^{1} \mathrm{H}$ NMR (DMSO- $\left.d_{6}\right) \delta \mathrm{ppm} 7.47(\mathrm{~m}, 6 \mathrm{H}, \mathrm{Ar}-\mathrm{H})$, $7.86(\mathrm{~m}, 5 \mathrm{H}, \mathrm{Ar}-\mathrm{H}), 8.47$ (s, 1H, Ar-H), 8.72 (s, 1H, Ar-H), 8.95 (s, $1 \mathrm{H}, \mathrm{N}=\mathrm{CH}), 9.09\left(\mathrm{~s}, 1 \mathrm{H}, \mathrm{CONH}, \mathrm{D}_{2} \mathrm{O}\right.$ exchangeable), $13.26(\mathrm{~s}, 1 \mathrm{H}$, $\mathrm{NH}, \mathrm{D}_{2} \mathrm{O}$ exchangeable); MS $\mathrm{m} / z: 409\left[(\mathrm{M}+1)^{+}, 8.18 \%\right], 408[(\mathrm{M}))^{+}$, 13.48\%], $288\left[\left(\mathrm{C}_{17} \mathrm{H}_{8} \mathrm{~N}_{2} \mathrm{O}_{3}\right)^{+}, 100 \%\right]$. Anal. Calcd. for $\mathrm{C}_{24} \mathrm{H}_{16} \mathrm{~N}_{4} \mathrm{O}_{3}: \mathrm{C}$, $67.91 ; \mathrm{H}, 3.80 ; \mathrm{N}, 13.20$. Found: C, 68.00; H, 3.60; N, 12.90 .

(ZE)6-(Benzo[d]oxazol-2-yl)-N'-(2-hydroxybenzylidene)-4oxo-1,4-dihydroquinoline-3-carbohydrazide (5b): Yield: 53\%; yellow solid; mp: $350-352^{\circ} \mathrm{C}$; IR $\left(\mathrm{cm}^{-1}\right): 3425-3141(\mathrm{OH}$ and $2 \mathrm{NH}), 3089(\mathrm{CH}$ aromatic), 1696, $1609(2 \mathrm{C}=\mathrm{O}) ;{ }^{1} \mathrm{H}$ NMR (DMSO- $\left.d_{6}\right) \delta \mathrm{ppm} 6.95(\mathrm{~m}$, 2H, Ar-H), 7.47 (m, 4H, Ar-H), 7.96 (m, 4H, Ar-H), 8.69 (s, 1H, Ar$\mathrm{H}), 8.95(\mathrm{~s}, 1 \mathrm{H}, \mathrm{Ar}-\mathrm{H}), 9.08(\mathrm{~s}, 1 \mathrm{H}, \mathrm{N}=\mathrm{CH}), 9.09$ (s, 1H, CONH, $\mathrm{D}_{2} \mathrm{O}$ exchangeable), 11.33 (s, 1H, OH, $\mathrm{D}_{2} \mathrm{O}$ exchangeable), $13.20(\mathrm{~s}, 1 \mathrm{H}, \mathrm{NH}$, $\mathrm{D}_{2} \mathrm{O}$ exchangeable); MS $\left.m / z: 425\left[(\mathrm{M}+1)^{+}, 3.52 \%\right], 424[(\mathrm{M}))^{+}, 5.37 \%\right]$, $80\left[\left(\mathrm{C}_{3} \mathrm{~N}_{2} \mathrm{O}\right)^{+}, 100 \%\right]$. Anal. Calcd. for $\mathrm{C}_{24} \mathrm{H}_{16} \mathrm{~N}_{4} \mathrm{O}_{4}: \mathrm{C}, 67.92 ; \mathrm{H}, 3.80 ; \mathrm{N}$, 13.20. Found: $\mathrm{C}, 67.80 ; \mathrm{H}, 3.50 ; \mathrm{N}, 13.00$.

(ZE)6-(Benzo[d]oxazol-2-yl)-N'-(4-florobenzylidene)-4-oxo1,4-dihydroquinoline-3-carbohydrazide (5c): Yield: 53\%; yellow solid; mp: $354-356^{\circ} \mathrm{C}$; IR ( $\left.\mathrm{cm}^{-1}\right): 3441,3141$ (2NH), 3088 (CH aromatic), 1696, $1623(2 \mathrm{C}=\mathrm{O})$; ${ }^{1} \mathrm{H}$ NMR (DMSO-d $) \delta \mathrm{ppm} 7.34(\mathrm{~m}, 2 \mathrm{H}, \mathrm{Ar}-\mathrm{H})$, 7.47 (m, 2H, Ar-H), 7.96 (m, 4H, Ar-H), 7.99 (m, 2H, Ar-H), 8.48 (s, $1 \mathrm{H}, \mathrm{Ar}-\mathrm{H}), 8.54$ (s, 1H, Ar-H), 8.95 (s, 1H, N=CH), 9.09 (s, 1H, CONH, $\mathrm{D}_{2} \mathrm{O}$ exchangeable), 13.26 (s, $1 \mathrm{H}, \mathrm{NH}, \mathrm{D}_{2} \mathrm{O}$ exchangeable); $\mathrm{MS} \mathrm{m} / z: 427$ $\left.\left[(\mathrm{M}+1)^{+}, 21.86 \%\right], 426[(\mathrm{M}))^{+}, 29.96 \%\right], 79\left[\left(\mathrm{C}_{5} \mathrm{~F}\right)^{+}, 100 \%\right]$. Anal. Calcd. For $\mathrm{C}_{24} \mathrm{H}_{15} \mathrm{FN}_{4} \mathrm{O}_{3}: \mathrm{C}, 67.60 ; \mathrm{H}, 3.55 ; \mathrm{N}, 13.14$. Found: $\mathrm{C}, 67.40 ; \mathrm{H}, 3.30$; $\mathrm{N}, 13.10$.

(ZE)6-(Benzo[d] oxazol-2-yl)-N'-(4-chlorobenzylidene)-4-oxo1,4-dihydroquinoline-3-carbohydrazide (5d): Yield: 53\%; yellow solid; mp: $378-380^{\circ} \mathrm{C}$; IR ( $\left.\mathrm{cm}^{-1}\right): 3430,3141(2 \mathrm{NH}), 3088$ (CH aromatic), 1696, $1623(2 \mathrm{C}=\mathrm{O}) ;{ }^{1} \mathrm{H}$ NMR (DMSO- $\left.d_{6}\right) \delta \mathrm{ppm} 7.47(\mathrm{~m}, 1 \mathrm{H}, \mathrm{Ar}-\mathrm{H})$, $7.68(\mathrm{~m}, 2 \mathrm{H}, \mathrm{Ar}-\mathrm{H}), 7.81$ (d, 2H, J= 8.4 Hz, Ar-H), 7.87 (m, 3H, Ar-H), 7.93 (m, 2H, Ar-H), 7.97 (s, 1H, Ar-H), 8.48 (s, 1H, Ar-H), 8.95 (s, $1 \mathrm{H}, \mathrm{N}=\mathrm{CH}), 9.09\left(\mathrm{~s}, 1 \mathrm{H}, \mathrm{CONH}, \mathrm{D}_{2} \mathrm{O}\right.$ exchangeable), $13.26(\mathrm{~s}, 1 \mathrm{H}, \mathrm{NH}$, $\mathrm{D}_{2} \mathrm{O}$ exchangeable); $\mathrm{MS} m / z: 443\left[\left.(\mathrm{M})\right|^{+}, 6.22 \%\right], 80\left[\left(\mathrm{C}_{3} \mathrm{~N}_{2} \mathrm{O}\right)^{+}, 100 \%\right]$. Anal. Calcd. For $\mathrm{C}_{24} \mathrm{H}_{15} \mathrm{ClN}_{4} \mathrm{O}_{3}: \mathrm{C}, 65.09 ; \mathrm{H}, 3.41 ; \mathrm{N}, 12.65$. Found: C, $64.80 ; \mathrm{H}, 3.10 ; \mathrm{N}, 12.70$.

(ZE)6-(Benzo[d]oxazol-2-yl)-N' - (4 (dimethylamino) benzylidene)-4-oxo-1,4-dihydroquinoline-3-carbohydrazide (5e): Yield: 53\%; orange solid; mp: $354-356^{\circ} \mathrm{C}$; IR $\left(\mathrm{cm}^{-1}\right): 3436,3141(2 \mathrm{NH})$, 3085 ( $\mathrm{CH}$ aromatic), 1696, $1600(2 \mathrm{C}=\mathrm{O}) ;{ }^{1} \mathrm{H}$ NMR (DMSO-d $)$ ) $\delta \mathrm{ppm}$ $3.6\left(\mathrm{~s}, 6 \mathrm{H}, 2 \mathrm{NCH}_{3}\right), 7.55(\mathrm{~m}, 6 \mathrm{H}, \mathrm{Ar}-\mathrm{H}), 8.26(\mathrm{~m}, 6 \mathrm{H}, \mathrm{Ar}-\mathrm{H}), 8.39(\mathrm{~s}$, $1 \mathrm{H}, \mathrm{CONH}, \mathrm{D}_{2} \mathrm{O}$ exchangeable), $8.93(\mathrm{~s}, 1 \mathrm{H}, \mathrm{N}=\mathrm{CH}), 14.26(\mathrm{~s}, 1 \mathrm{H}, \mathrm{NH}$, $\mathrm{D}_{2} \mathrm{O}$ exchangeable); $\mathrm{MS} \mathrm{m} / z: 451\left[(\mathrm{M})^{++}, 9.64 \%\right], 80\left[\left(\mathrm{C}_{5} \mathrm{H}_{6} \mathrm{~N}\right)^{+}, 100 \%\right]$. Anal. Calcd. For $\mathrm{C}_{26} \mathrm{H}_{21} \mathrm{~N}_{5} \mathrm{O}_{3}$ : C, 69.17; H, 4.69; N, 15.51. Found: C, $68.80 ; \mathrm{H}, 4.40 ; \mathrm{N}, 15.50$.

(ZE)6-(Benzo[d]oxazol-2-yl)-N'-(4-nitrobenzylidene)-4-oxo- 
Citation: Abdelgawad MA, Lamie PF, Ahmed OM (2016) Synthesis of New Quinolone Derivatives Linked to Benzothiazole or Benzoxazole Moieties as Anticancer and Anti-Oxidant Agents. Med Chem (Los Angeles) 6: 652-657. doi:10.4172/2161-0444.1000410

1,4-dihydroquinoline-3-carbohydrazide (5f): Yield: 53\%; brown solid; $\mathrm{mp}$ : $347-349^{\circ} \mathrm{C}$; IR $\left(\mathrm{cm}^{-1}\right): 3434,3141(2 \mathrm{NH}), 3089$ ( $\mathrm{CH}$ aromatic), 1694, $1617(2 \mathrm{C}=\mathrm{O}) ;{ }^{1} \mathrm{H}$ NMR (DMSO- $\left.d_{6}\right) \delta \mathrm{ppm} 7.54(\mathrm{~m}, 4 \mathrm{H}, \mathrm{Ar}-$ $\mathrm{H}), 7.76(\mathrm{~m}, 2 \mathrm{H}, \mathrm{Ar}-\mathrm{H}), 8.13(\mathrm{~m}, 3 \mathrm{H}, \mathrm{Ar}-\mathrm{H}), 8.82(\mathrm{~s}, 2 \mathrm{H}, \mathrm{N}=\mathrm{CH}$ and CONH, $\mathrm{D}_{2} \mathrm{O}$ exchangeable), $8.87(\mathrm{~m}, 3 \mathrm{H}, \mathrm{Ar}-\mathrm{H}), 11.19\left(\mathrm{~s}, 1 \mathrm{H}, \mathrm{NH}, \mathrm{D}_{2} \mathrm{O}\right.$ exchangeable); MS $\left.m / z: 454\left[(\mathrm{M}+1)^{+}, 23.62 \%\right], 453[(\mathrm{M}))^{+}, 40.94 \%\right]$, $61\left[\left(\mathrm{C}_{5} \mathrm{H}\right)^{+}, 100 \%\right]$. Anal. Calcd. For $\mathrm{C}_{24} \mathrm{H}_{15} \mathrm{~N}_{5} \mathrm{O}_{5}: \mathrm{C}, 63.58 ; \mathrm{H}, 3.33 ; \mathrm{N}$, 15.45. Found: C, 63.20; H, 3.00; N, 15.20.

(ZE)6-(Benzo[d] thiazol-2-yl)-N'-benzylidene-4-oxo-1,4dihydroquinoline-3-carbohydrazide (5g): Yield: $53 \%$; yellow solid; mp: 396-398 ${ }^{\circ} \mathrm{C}$; IR $\left(\mathrm{cm}^{-1}\right)$ : 3404, $3259(2 \mathrm{NH}), 3061(\mathrm{CH}$ aromatic), 1661, $1622(2 \mathrm{C}=\mathrm{O})$; ${ }^{1} \mathrm{H}$ NMR (DMSO- $\left.d_{6}\right) \delta \mathrm{ppm} 7.45(\mathrm{~m}, 4 \mathrm{H}, \mathrm{Ar}-$ $\mathrm{H}), 7.56(\mathrm{~m}, 2 \mathrm{H}, \operatorname{Ar}-\mathrm{H}), 7.78(\mathrm{~m}, 2 \mathrm{H}, \mathrm{Ar}-\mathrm{H}), 8.16(\mathrm{~m}, 2 \mathrm{H}, \mathrm{Ar}-\mathrm{H})$, $8.79(\mathrm{~s}, 2 \mathrm{H}, \mathrm{Ar}-\mathrm{H}), 8.80(\mathrm{~s}, 1 \mathrm{H}, \mathrm{N}=\mathrm{CH}), 8.91(\mathrm{~d}, 1 \mathrm{H}, \mathrm{J}=14.8 \mathrm{~Hz}, \mathrm{Ar}-$ $\mathrm{H}), 10.87\left(\mathrm{~s}, 1 \mathrm{H}, \mathrm{CONH}, \mathrm{D}_{2} \mathrm{O}\right.$ exchangeable), $14.02\left(\mathrm{~s}, 1 \mathrm{H}, \mathrm{NH}, \mathrm{D}_{2} \mathrm{O}\right.$ exchangeable); MS $m / z: 425$ [(M+1)+, 43.94\%], $424\left[\left.(\mathrm{M})\right|^{+}, 53.03 \%\right]$, $55\left[\left(\mathrm{C}_{4} \mathrm{H}_{7}\right)^{+}, 100 \%\right]$. Anal. Calcd. For $\mathrm{C}_{24} \mathrm{H}_{16} \mathrm{~N}_{4} \mathrm{O}_{2} \mathrm{~S}: \mathrm{C}, 67.91 ; \mathrm{H}, 3.80 ; \mathrm{N}$, 13.20. Found: C, 67.70; H, 3.80; N, 13.10 .

(ZE)6-(Benzo[d] thiazol-2-yl)-N'-(2-hydroxybenzylidene)-4oxo-1,4-dihydroquinoline-3-carbohydrazide (5h): Yield: $48 \%$; yellow solid; mp: $362-364^{\circ} \mathrm{C}$; IR $\left(\mathrm{cm}^{-1}\right): 3400-3166(\mathrm{OH}$ and $2 \mathrm{NH}), 3057(\mathrm{CH}$ aromatic), 1658, $1617(2 \mathrm{C}=\mathrm{O}) ;{ }^{1} \mathrm{H}$ NMR (DMSO- $\left.d_{6}\right) \delta \mathrm{ppm} 6.96(\mathrm{~m}$, 2H, Ar-H), $7.54(\mathrm{~m}, 4 \mathrm{H}, \operatorname{Ar}-\mathrm{H}), 7.84(\mathrm{~m}, 1 \mathrm{H}, \mathrm{Ar}-\mathrm{H}), 8.09$ (d, 2H, J= $1.2 \mathrm{~Hz}, \mathrm{Ar}-\mathrm{H}), 8.40$ (m, 1H, A-H), 8.62 (s, 1H, N=CH), 8.90 (s, 2H, Ar$\mathrm{H}), 10.69$ (s, 1H, CONH, $\mathrm{D}_{2} \mathrm{O}$ exchangeable), 11.46 (s, 1H, OH, $\mathrm{D}_{2} \mathrm{O}$ exchangeable), 13.78 (s, $1 \mathrm{H}, \mathrm{NH}, \mathrm{D}_{2} \mathrm{O}$ exchangeable) ; MS $m / z: 441$ $\left[(\mathrm{M}+1)^{+}, 61.22 \%\right], 440\left[(\mathrm{M})^{+}+, 56.12 \%\right], 135\left[\left(\mathrm{C}_{7} \mathrm{H}_{7} \mathrm{~N}_{2} \mathrm{O}\right)^{+}, 100 \%\right]$. Anal. Calcd. For $\mathrm{C}_{24} \mathrm{H}_{16} \mathrm{~N}_{4} \mathrm{O}_{3} \mathrm{~S}$ : C, 65.44; H, 3.66; N, 12.72. Found: C, 65.60; $\mathrm{H}, 3.40 ; \mathrm{N}, 12.50$.

(ZE)6-(Benzo[d] thiazol-2-yl)-N'-(4-flurobenzylidene)-4oxo-1,4-dihydroquinoline-3-carbohydrazide (5i): Yield: $48 \%$; yellow solid; mp: $395-397^{\circ} \mathrm{C}$; IR $\left(\mathrm{cm}^{-1}\right): 3426,3262(2 \mathrm{NH}), 3063(\mathrm{CH}$ aromatic), 1661, $1623(2 \mathrm{C}=\mathrm{O}) ;{ }^{1} \mathrm{H}$ NMR (DMSO- $\left.d_{6}\right) \delta \mathrm{ppm} 7.54(\mathrm{~m}$, $4 \mathrm{H}, \mathrm{Ar}-\mathrm{H}), 8.08$ (m, 2H, Ar-H), $8.13(\mathrm{~m}, 3 \mathrm{H}, \mathrm{Ar}-\mathrm{H}), 8.84(\mathrm{~m}, 2 \mathrm{H}, \mathrm{Ar}-$ $\mathrm{H}), 9.60(\mathrm{~s}, 1 \mathrm{H}, \mathrm{N}=\mathrm{CH}), 9.61(\mathrm{~s}, 1 \mathrm{H}, \mathrm{Ar}-\mathrm{H}), 11.00\left(\mathrm{~s}, 1 \mathrm{H}, \mathrm{CONH}, \mathrm{D}_{2} \mathrm{O}\right.$ exchangeable), $13.26\left(\mathrm{~s}, 1 \mathrm{H}, \mathrm{NH}, \mathrm{D}_{2} \mathrm{O}\right.$ exchangeable); MS $m / z: 443$ $\left[(\mathrm{M}+1)^{+}, 3.21 \%\right], 442\left[(\mathrm{M})^{+}+, 21.57 \%\right], 69\left[\left(\mathrm{C}_{5} \mathrm{H}_{9}\right)^{+}, 100 \%\right]$. Anal. Calcd. For $\mathrm{C}_{24} \mathrm{H}_{15} \mathrm{FN}_{4} \mathrm{O}_{2} \mathrm{~S}: \mathrm{C}, 65.15 ; \mathrm{H}, 3.42 ; \mathrm{N}, 12.66$. Found: C, 64.90; $\mathrm{H}, 3.20$; $\mathrm{N}, 12.70$.

(ZE)6-(Benzo[d] thiazol-2-yl)-N'-(4-chlorobenzylidene)-4oxo-1,4-dihydroquinoline-3-carbohydrazide (5j): Yield: 48\%; yellow solid; mp: $398-400^{\circ} \mathrm{C}$; IR $\left(\mathrm{cm}^{-1}\right): 3432,3209(2 \mathrm{NH}), 3047(\mathrm{CH}$ aromatic), 1660, $1621(2 \mathrm{C}=\mathrm{O}) ;{ }^{1} \mathrm{H}$ NMR (DMSO-d $\left.{ }_{6}\right) \delta \mathrm{ppm} 7.53(\mathrm{~m}$, $4 \mathrm{H}, \mathrm{Ar}-\mathrm{H}), 7.77$ (m, 3H, Ar-H), $7.79(\mathrm{~m}, 4 \mathrm{H}, \mathrm{Ar}-\mathrm{H}), 8.38$ (s, 1H, Ar-H), $8.91(\mathrm{~s}, 1 \mathrm{H}, \mathrm{N}=\mathrm{CH}), 10.08$ (s, $1 \mathrm{H}, \mathrm{CONH}, \mathrm{D}_{2} \mathrm{O}$ exchangeable), 14.41 (s, $1 \mathrm{H}, \mathrm{NH}, \mathrm{D}_{2} \mathrm{O}$ exchangeable $) ; \mathrm{MS} m / z: 459$ [(M) $\left.{ }^{+}, 19.07 \%\right], 458$ [(M$\left.\mathrm{H})^{+}, 17.53 \%\right], 54\left[\left(\mathrm{C}_{4} \mathrm{H}_{6}\right)^{+}, 100 \%\right]$. Anal. Calcd. For $\mathrm{C}_{24} \mathrm{H}_{15} \mathrm{ClN}_{4} \mathrm{O}_{2} \mathrm{~S}: \mathrm{C}$, $62.81 ; \mathrm{H}, 3.29 ; \mathrm{N}, 12.21$. Found: C, 62.60; H, 3.40; N, 12.10 .

(ZE)6-(Benzo[d] thiazol-2-yl)-N'-(4-(dimethylamino) benzylidene)-4-oxo-1,4-dihydroquinoline-3-carbohydrazide (5k): Yield: $48 \%$; orange solid; mp: $378-380^{\circ} \mathrm{C}$; IR $\left(\mathrm{cm}^{-1}\right): 3420,3263(2 \mathrm{NH})$, 3065 (CH aromatic), 1661, $1622(2 \mathrm{C}=\mathrm{O}){ }^{1} \mathrm{H}$ NMR (DMSO- $\left.\mathrm{d}_{6}\right) \delta \mathrm{ppm}$ $3.45\left(\mathrm{~s}, 6 \mathrm{H}, 2 \mathrm{CH}_{3}\right), 7.00(\mathrm{~d}, 2 \mathrm{H}, J=4.4 \mathrm{~Hz}, \mathrm{Ar}-\mathrm{H}), 7.49(\mathrm{~m}, 4 \mathrm{H}, \mathrm{Ar}-\mathrm{H})$, $7.54(\mathrm{~m}, 2 \mathrm{H}, \mathrm{Ar}-\mathrm{H}), 8.16(\mathrm{~d}, 1 \mathrm{H}, J=7.6 \mathrm{HZ}, \mathrm{Ar}-\mathrm{H}), 8.34(\mathrm{~d}, 1 \mathrm{H}, J=8.4$ $\mathrm{Hz}, \mathrm{Ar}-\mathrm{H}), 8.81$ (s, 1H, Ar-H), 8.90 (s, 1H, N=CH), 8.92 (s, 1H, ArH) $10.96\left(\mathrm{~s}, 1 \mathrm{H}, \mathrm{CONH}, \mathrm{D}_{2} \mathrm{O}\right.$ exchangeable), $14.22\left(\mathrm{~s}, 1 \mathrm{H}, \mathrm{NH}, \mathrm{D}_{2} \mathrm{O}\right.$ exchangeable); MS $m / z: 468\left[(\mathrm{M}+1)^{++}, 25 \%\right], 467\left[\left.(\mathrm{M})\right|^{++}, 7.41 \%\right], 217$ $\left[\left(\mathrm{C}_{12} \mathrm{H}_{15} \mathrm{~N}_{3} \mathrm{O}\right)^{+}\right.$, 100\%]. Anal. Calcd. For $\mathrm{C}_{26} \mathrm{H}_{21} \mathrm{~N}_{5} \mathrm{O}_{2} \mathrm{~S}: \mathrm{C}, 66.79 ; \mathrm{H}, 4.53$; $\mathrm{N}, 14.98$. Found: $\mathrm{C}, 66.90 ; \mathrm{H}, 4.40 ; \mathrm{N}, 14.90$.
(ZE)6-(Benzo[d]thiazol-2-yl)-N'-(4-nitrobenzylidene)-4-oxo1,4-dihydroquinoline-3-carbohydrazide (51): Yield: 48\%;brown solid; mp: $356-358^{\circ} \mathrm{C}$; IR $\left(\mathrm{cm}^{-1}\right): 3419,3212(2 \mathrm{NH}), 3160,3053(\mathrm{CH}$ aromatic), 1664, $1623(2 \mathrm{C}=\mathrm{O}) ;{ }^{1} \mathrm{H}$ NMR (DMSO- $\left.d_{6}\right) \delta \mathrm{ppm} 7.48(\mathrm{~m}$, $3 \mathrm{H}, \mathrm{Ar}-\mathrm{H}), 8.16(\mathrm{~m}, 7 \mathrm{H}, \mathrm{Ar}-\mathrm{H}), 8.41(\mathrm{~s}, 1 \mathrm{H}, \mathrm{Ar}-\mathrm{H}), 8.92(\mathrm{~s}, 1 \mathrm{H}, \mathrm{N}=\mathrm{CH})$, 8.93 (s, 2H, Ar-H and CONH, D O exchangeable), $14.62(\mathrm{~s}, 1 \mathrm{H}, \mathrm{NH}$, $\mathrm{D}_{2} \mathrm{O}$ exchangeable) ; $\mathrm{MS} m / z: 470\left[(\mathrm{M}+1)^{++}, 23.77 \%\right], 469\left[\left.(\mathrm{M})\right|^{+}\right.$, 60.66\%], $428\left[\left(\mathrm{C}_{23} \mathrm{H}_{14} \mathrm{~N}_{3} \mathrm{O}_{4} \mathrm{~S}\right)^{+}, 100 \%\right]$. Anal. Calcd. For $\mathrm{C}_{24} \mathrm{H}_{15} \mathrm{~N}_{5} \mathrm{O}_{4} \mathrm{~S}$ : C, $61.40 ; \mathrm{H}, 3.22 ; \mathrm{N}, 14.92$. Found: C, $61.20 ; \mathrm{H}, 3.50 ; \mathrm{N}, 14.70$.

\section{Biological Evaluation}

\section{Anticancer screening}

Human tumor cell lines: Breast carcinoma cell lines (MCF-7) used in this study were obtained from the American Type Culture Collection (ATCC, Minisota, USA) through the Tissue Culture Unit, the Egyptian Organization for Biological Products and Vaccines, Vacsera, 51 Wezaret EI Zeraa St., Agouza, Giza, Egypt. The tumor cell lines were maintained at Center for Genetic Engineering, Al-Azhar University, Cairo, Egypt by serial sub-culturing.

\section{Chemicals}

Dimethylsulphoxide (DMSO), Dulbecco's Modified Eagle Medium (DMEM), trypan blue, Fetal Bovine Serum, Penicillin/ Streptomycin antibiotic and Trypsin- EDTA Sigma Aldrich Chemical Co., St. Louis, Mo, USA. Tris buffer was obtained from Applichem, Germany. All chemicals and reagents used in this study are of highest analytical grade.

\section{Methods}

\section{Preparation of test compounds}

The tested derivatives $\mathbf{5 a - 1}$ were prepared by dissolving in dimethylsulfoxide (DMSO) and the prepared stock was stored at $-20^{\circ} \mathrm{C}$. Different concentrations of the compounds $0,6.25,12.5,25,50$ and 100 $\mu \mathrm{g} / \mathrm{ml}$ in culture medium were used.

\section{Preparatory steps prior to cytotoxicity investigation}

Maintenance of the breast carcinoma cell lines (MCF-7) in the laboratory, cryopreservation of cells, collection of cells by trypsinization and determination and counting of viable cells are performed according to the reported methods $[20,21]$.

\section{Determination of potential cytotoxicity of drug on human cancer cell line}

The cytotoxicity was carried out using Sulphorhodamine-B (SRB) assay following the reported method [22] SRB is a bright pink aminoxanthrene dye with two sulphonic groups. It is a protein stain that binds to the amino groups of intracellular proteins under mildly acidic conditions to provide a sensitive index of cellular protein content.

Cells of MCF-7 cell lines are seeded in 96 well microliter plates at a concentration of 1000-2000 cells/well, $100 \mu \mathrm{l} /$ well. After $24 \mathrm{~h}$, cells will be incubated for $72 \mathrm{~h}$ with various concentrations of drugs $(0$, $6.25,12.5,25,50$ and $100 \mu \mathrm{g} / \mathrm{ml})$. Dulbecco's Modified Eagle Medium (DMEM) with $10 \%$ foetal calf serum, sodium pyruvate, $100 \mathrm{U} / \mathrm{ml}$ penicillin and $100 \mathrm{mg} / \mathrm{ml}$ streptomycin at $37^{\circ} \mathrm{C}$ and $5 \% \mathrm{CO}_{2}$, was used as culture medium. At the end of the incubation, the medium is discarded. The cells are fixed with $150 \mu$ l cold trichloroacetic acid $10 \%$ final concentration for 1 hour at $4^{\circ} \mathrm{C}$. The plates were washed with distilled water using (automatic washer Tecan, Germany) and stained 
with $50 \mu \mathrm{l} 0.4 \%$ SRB dissolved in $1 \%$ acetic acid for 30 minutes at room temperature in dark. The plates were washed with $1 \%$ acetic acid to remove unbound dye and air-dried $(24 \mathrm{~h})$. The dye was solubilized with $150 \mu \mathrm{l} /$ well of $10 \mathrm{mMtris}$ base (PH 7.4) for $5 \mathrm{~min}$ on a shaker at $1600 \mathrm{rpm}$. The optical density (OD) of each well will be measured spectrophotometrically at $490 \mathrm{~nm}$ with an ELISA microplate reader. The mean background absorbance was automatically subtracted and mean values of each derivative and 4-(benzothiazol-2-yl) aniline (reference drug) concentration was calculated. The experiment was repeated three times. The percentage of cell survival was calculated by using formula, surviving percent $=[\mathrm{OD}$ (treated cells) $/ \mathrm{OD}$ (control cells) $] \times 100$. The $\mathrm{IC}_{50}$ values (the concentrations of derivatives required to produce $50 \%$ inhibition of cell growth) were also calculated using linear trend linear equation.

\section{Anti-oxidant assay}

DPPH radical scavenging activity: The effect of the synthesized organic compounds on DPPH radicalwas estimated using the reported methods [23,24] with some modifications. A solution of $200 \mu \mathrm{mol}$ DPPH in ethanol was prepared and $100 \mu \mathrm{l}$ of this solution was mixed with $0.9 \mathrm{ml}$ of varying concentrations of the synthesized derivatives (dissolved in ethanol) to reach a final concentration of $0.25,0.5$ and $1 \mathrm{mg} / \mathrm{ml}$. The reaction mixture wasvortexed and left in the dark for $30 \mathrm{~min}$ (room temperature). The color became light yellow from deep violet and the absorbanceof the mixture was determined at $570 \mathrm{~nm}$. The control was prepared by using adding $100 \mu \mathrm{l} \mathrm{DPPH}$ to $0.9 \mathrm{ml}$ ethanol solution. 100

DPPH radical scavenging activity $(\%)=1 / 2\left[\left(\mathrm{~A}_{\text {control }}-\mathrm{A}_{\text {sample }}\right) / \mathrm{A}_{\text {control }}\right] \times$

Where $\mathrm{A}_{\text {control }}$ is the absorbance of DPPH radical+ethanol and $\mathrm{A}_{\text {sample }}$ is the absorbance of DPPH radical+sample of derivative compound dissolved in ethanol.

\section{Results and Discussion}

\section{Chemistry}

In this work, the synthesis of different Schiffbases at $C$-3 of quinolone moiety was described. Biologically important benzoxazoles and benzothiazoles were merged with quinolone nucleus. 6-Benzoxazole/ benzothiazol-2-yl-4-oxo-1,4-dihydro-quinoline-3-carboxylic acid ethyl esters $\mathbf{3} \mathbf{a} \& \mathbf{b}$ were employed as the starting materials. They were synthesized following the precautions of their reported method [14]. Direct conversion of $\mathbf{3} \mathbf{a} \& \mathbf{b}$ into hydrazide derivatives $4 \mathbf{a} \& \mathbf{b}$ was achieved in good yield by the treatment of $\mathbf{3 a} \mathbf{a} \mathbf{b}$ with hydrazine hydrate $99 \%$. Several solvents were carried out to prepare these intermediates $\mathbf{4} \mathbf{a} \& \mathbf{b}$ such as absolute ethanol, glacial acetic acid and dioxane. Absolute ethanol was the solvent of choice since it gave more pure products (using: TLC) as well as higher yields (Scheme 1). The structure of compounds $\mathbf{4 a \& b}$ was established on the basis of IR, ${ }^{1} \mathrm{H}$ NMR, mass spectral data and elemental analysis. IR spectra of $\mathbf{4 a \& b}$ revealed the appearance of new absorption bands at $3465-3176 \mathrm{~cm}^{-1}$ due to $2 \mathrm{NH}$ and $\mathrm{NH}_{2}$ groups.

The structure of the compounds $\mathbf{4 a \& b}$ was confirmed by ${ }^{1} \mathrm{H}$ NMR which was performed in DMSO as a solvent, appearance of $\mathrm{D}_{2} \mathrm{O}$ exchangeable singlet signal at $\delta 4.51$ and $4.65 \mathrm{ppm}$ indicated $\mathrm{NH}_{2}$ protons, beside the appearance of another exchangeable singlet signal corresponding to $\mathrm{CONH}$ proton at $\delta 10.63$ and $10.84 \mathrm{ppm}$, respectively. Moreover, mass spectra of $\mathbf{4} \mathbf{a} \& \mathbf{b}$ showed molecular ion peaks at $\mathrm{m} / z$ 320 and 336, sequentially. Heating compounds $4 \mathbf{a} \& \mathbf{b}$ for 8-10 hours with different aromatic aldehydes in absolute ethanol containing catalytic amount of glacial acetic acid led to the formation of $\mathbf{5 a - 1}$. The formation of the target Schiff bases 5a-1 was substantiated on basis of spectral data and elemental analysis (see Experimental Section).

${ }^{1} \mathrm{H}$ NMR spectra of compounds 5a-1 showed disappearance of $\mathrm{D}_{2} \mathrm{O}$ exchangeable singlet signal of $\mathrm{NH}_{2}$ protons of the parent hydrazide derivatives $\mathbf{4 a \& b}$ and the appearance of new singlet signal due to azomethine proton $(\mathrm{N}=\mathrm{CH})$ at $\delta 8.82-9.08 \mathrm{ppm}$ in benzoxazole derivatives 5a-f or at $\delta 8.80-9.60$ ppm for benzothiazole derivatives $\mathbf{5 g - 1}$.

Additionally, the mass spectrum of compound 5d reveled molecular ion peaks at $\mathrm{m} / \mathrm{z} 443$ and 445 corresponding to $(\mathrm{M})^{+}$and $(\mathrm{M}+2)^{+}$, respectively in ratio of $3: 1(\mathrm{Cl}$ pattern). The reactivity of the applied aromatic aldehydes was appeared in parallel manner with the yield of the resulting targets $\mathbf{5 a - 1}$, which ranged between $45 \%$ up to $79 \%$ starting from benzaldehyde to 4-nitrobenzaldehyde in both benzoxazole and benzothiazole derivatives.

\section{Anticancer activity}

The data showing the anti-proliferative effects of the tested derivatives on breast carcinoma cell lines (MCF-7) are illustrated in Figures 1 and 2. All derivatives from $\mathbf{5 a}$ to $\mathbf{5 l}$ produced a marked gradual decrease in the survival percent of MCF-7 as the dose of derivatives increased from 0 to $100 \mu \mathrm{g} / \mathrm{ml}$. Based on the values of $\mathrm{IC}_{50}$, the derivatives are arranged according to their tumor cytotoxic potencies in the following order: derivatives $\mathbf{5 l}, \mathbf{5 h}, \mathbf{5 g}, \mathbf{5 k}, \mathbf{5 e}, \mathbf{5 f}, \mathbf{5 a}$, $\mathbf{5 i}, \mathbf{5 d}, \mathbf{5 j}, \mathbf{5 c}$ and $\mathbf{5 a}$ recording $\mathrm{IC}_{50}$ of $40.783,45.461,48.953,50.627$, $52.138,53.665,54.163,55.344,57.462,58.452,69.214$ and $82.300 \mu \mathrm{g} / \mathrm{ml}$, respectively Scheme 2 . Thus, derivative 51 produced the most potent tumor cytotoxic efficacy, while derivative $\mathbf{5 a}$ followed by derivative $\mathbf{5 c}$ are the least potent (Figure 2 and Table 1).

\section{Anti-oxidant activity}

The antioxidant capacity was evaluated by detection of DPPH radical scavenging activity. Different concentrations of derivatives were tested. All tested derivatives had marked antioxidant activity. At
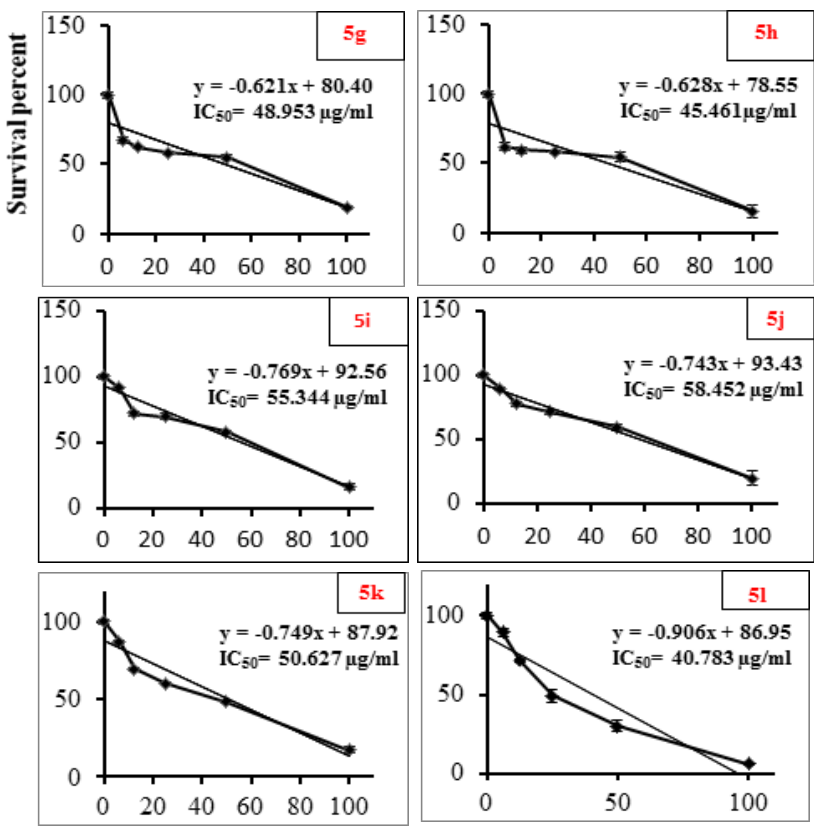

Concentration $(\mu \mathrm{g} / \mathrm{ml})$

Figure 2: Chemical structure of the target compounds 5a-I. 


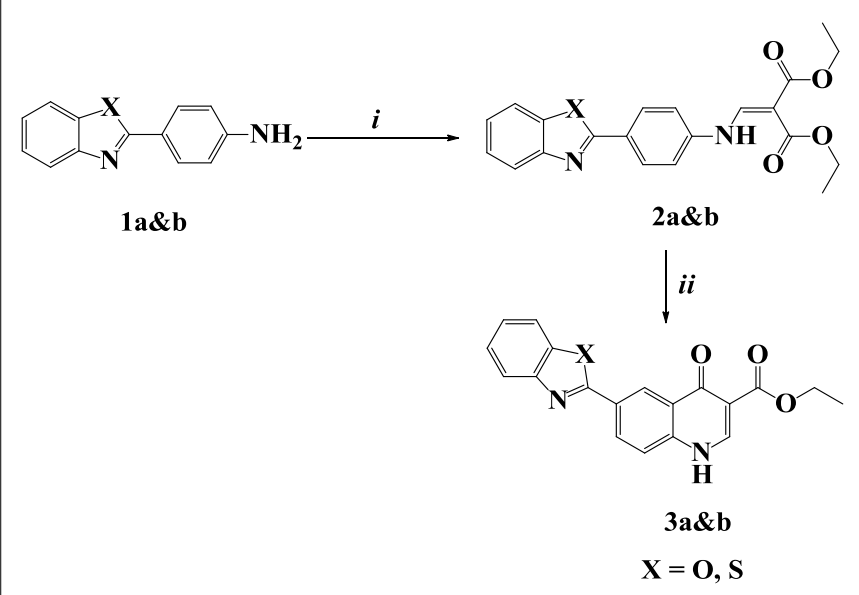

Scheme 1: Reagents and conditions: i) EMME, ethanol reflux for $4 \mathrm{~h}$; ii) diphenyl ether, reflux for $1 \mathrm{~h}$.

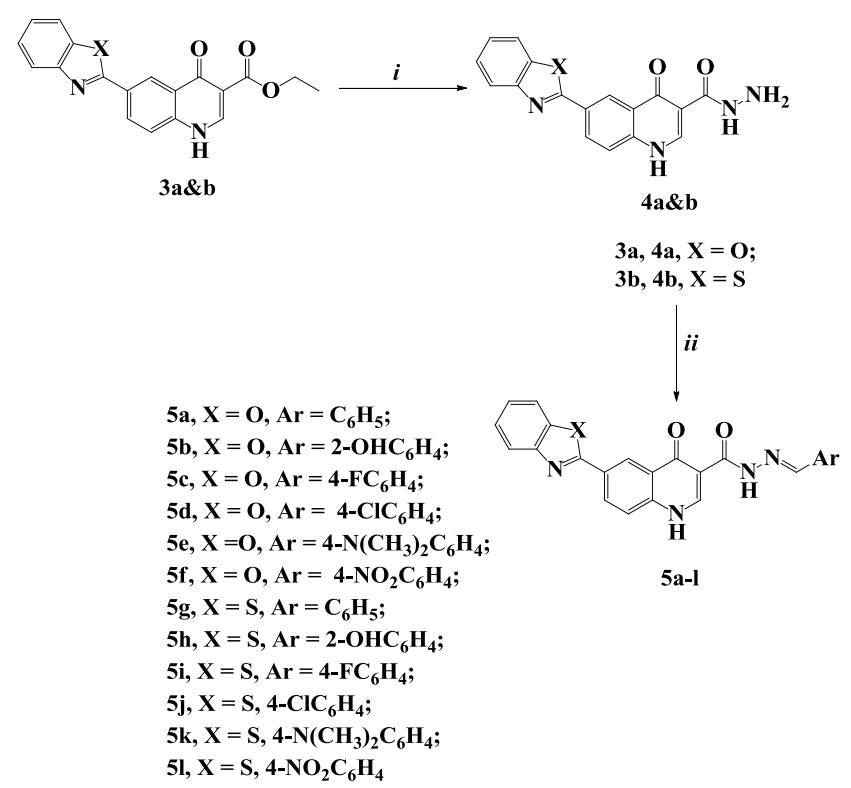

Scheme 2: Reagents and conditions: i) $\mathrm{N}_{2} \mathrm{H}_{4}$ absolute ethanol; ii) $\mathrm{ArCHO}$ absolute ethanol, drops of glacial acetic acid.

lowest dose, $0.25 \mathrm{mg} / \mathrm{ml}$, derivative $\mathbf{5 a}$ followed by $5 \mathbf{e}$ seemed to have the most potent DPPH radical scavenging activity percent recording 26.33 and $21.58 \%$, respectively. At medium dose $(0.5 \mathrm{mg} / \mathrm{ml})$ and high dose $(1 \mathrm{mg} / \mathrm{ml})$, derivatives $\mathbf{5 e}, \mathbf{5} \mathbf{g}$ and $\mathbf{5 h}$ were the most efficient in having antioxidant activity. Derivative $\mathbf{5 c}$ had no antioxidant activity at high dose although it had marked efficacy at low and medium doses. Moreover, while the antioxidant activity of $\mathbf{5 a}, \mathbf{5 b}$ and $\mathbf{5 l}$ was more or less unchanged as the dose increased from 0.5 to $1 \mathrm{mg} / \mathrm{ml}$, it was more potentiated for derivatives $\mathbf{5 d}, \mathbf{5 f}, \mathbf{5 g}, \mathbf{5 h}, \mathbf{5 i}, \mathbf{5 j}$ and $\mathbf{5 k}$ (Table 2).

\section{Conclusion}

The synthesized derivatives exhibited various degrees of cytotoxic effects on breast carcinoma cell line (MCF-7) in vitro. Comparing the new compounds with 4-(benzothiazol-2-yl)aniline - known with its cytotoxic activity- which has $\mathrm{IC}_{50} 0.065 \mu \mathrm{M}$, we observed that: (ZE)-6-(bnzo[d] thiazol-2-yl)-N'-(4-nitrobenzylidene)-4-oxo-1,4dihydroquinoline-3-carbohydrazide (5l) and (ZE)-6-(Benzo[d] thiazol-
2-yl)-N'-(2-hydroxybenzylidene)-4-oxo-1,4-dihydroquinoline-3carbohydrazide (5h) seemed to have the most potent antitumor action ( $\mathrm{IC}_{50}: 0.052 \mu \mathrm{M}$ and $0.058 \mu \mathrm{M}$, respectively), in addition to five new compounds $5 \mathbf{e}, \mathbf{5 f}, \mathbf{5 g}, \mathbf{5 i}$ and $5 \mathbf{k}$ showed good activity with $\mathrm{IC}_{50}$ between $0.072 \mu \mathrm{M}$ and $0.099 \mu \mathrm{M}$. Only five compounds $(\mathbf{5 a}, \mathbf{5 b}, \mathbf{5} \mathbf{c}, \mathbf{5 d}, \mathbf{5 j})$ exhibit moderate activity. with $\mathrm{IC}_{50}$ in the range of $0.10 \mu \mathrm{M}$ and $0.20 \mathrm{w} \mu \mathrm{M}$. On the other hand, (ZE)-6-(benzo[d] oxazol-2-yl)-N'-(4-(dimethylamino)benzylidene)-4-oxo-1,4dihydroquinoline-3-carbohydrazide (5e), (ZE)-6-(benzo[d]thiazol2-yl)-N'-benzylidene-4-oxo-1,4-dihydroquinoline-3-carbohydrazide (5g) and (ZE)-6-(benzo[d]thiazol-2-yl)-N'-(2-hydroxybenzylidene)4-oxo-1,4-dihydroquinoline-3-carbohydrazide (5h) have the most efficient antioxidant activity as indicated by the results of DPPH radical scavenging capacity. Finally, it was found that compound $\mathbf{5 h}$ bearing 4-hydroxyphenyl moiety and benzothiazole nucleus has dual anticancer and anti-oxidant activity and need further investigations.

\section{Conflict of Interest}

The authors declared that there is no conflict of interest.

\begin{tabular}{|c|c|}
\hline Compound No. & IC $_{\mathbf{5 0}}$ (uM) \\
\hline $\mathbf{5 a}$ & 0.2 \\
\hline $\mathbf{5 b}$ & 0.117 \\
\hline $\mathbf{5 c}$ & 0.15 \\
\hline $\mathbf{5 d}$ & 0.106 \\
\hline $\mathbf{5 e}$ & 0.078 \\
\hline $\mathbf{5 f}$ & 0.085 \\
\hline $\mathbf{5 g}$ & 0.072 \\
\hline $\mathbf{5 h}$ & 0.058 \\
\hline $\mathbf{5 i}$ & 0.099 \\
\hline $\mathbf{5 j}$ & 0.104 \\
\hline $\mathbf{5 k}$ & 0.074 \\
\hline $\mathbf{5 l}$ & 0.052 \\
\hline $\mathbf{4}$ & 0.065 \\
\hline
\end{tabular}

Table 1: $I_{50}$ of the test compounds (5a-I) against breast cancer (MCF-7).

\begin{tabular}{|c|c|c|c|}
\hline Derivatives & $\begin{array}{c}\mathbf{0 . 2 5} \\
\mathbf{m g} / \mathbf{m l}\end{array}$ & $\begin{array}{c}\mathbf{0 . 5} \\
\mathbf{m g} / \mathbf{m l}\end{array}$ & $\begin{array}{c}\mathbf{1} \\
\mathbf{m g} / \mathbf{m l}\end{array}$ \\
\hline $\mathbf{5 a}$ & 26.33 & 12.16 & 13.50 \\
\hline $\mathbf{5 b}$ & 15.58 & 10.75 & 11.50 \\
\hline $\mathbf{5 c}$ & 10.25 & 20.00 & 0.00 \\
\hline $\mathbf{5 d}$ & 15.75 & 13.75 & 18.33 \\
\hline $\mathbf{5 e}$ & 21.58 & 31.84 & 39.75 \\
\hline $\mathbf{5 f}$ & 3.58 & 2.5 & 9.83 \\
\hline $\mathbf{5 g}$ & 12.97 & 25.52 & 40.83 \\
\hline $\mathbf{5 h}$ & 12.97 & 25.52 & 40.82 \\
\hline $\mathbf{5 i}$ & 13.64 & 19.39 & 31.65 \\
\hline $\mathbf{5 j}$ & 8.92 & 6.58 & 11.75 \\
\hline $\mathbf{5 k}$ & 11.59 & 20.63 & 27.28 \\
\hline $\mathbf{5 l}$ & 12.33 & 14.83 & 15.92 \\
\hline
\end{tabular}

Table 2: DPPH radical scavenging activity (\%) of derivative compounds (5a-I) at various concentrations. 
Citation: Abdelgawad MA, Lamie PF, Ahmed OM (2016) Synthesis of New Quinolone Derivatives Linked to Benzothiazole or Benzoxazole Moieties as Anticancer and Anti-Oxidant Agents. Med Chem (Los Angeles) 6: 652-657. doi:10.4172/2161-0444.1000410

\section{References}

1. Sissi C, Palumbo M (2003) The quinolone family: from antibacterial to anticancer agents. Curr Med Chem Anticancer agents 3: 439-450.

2. Orhan PM, Tekiner B, Suzen S (2013) Recent studies of antioxidant quinoline derivatives. Mini Rev Med Chem 13: 365-372.

3. Lucielli S, Aline IV, Natália S, Bruna SG, Micheli RC, et al. (2013) Synthesis and antioxidant properties of novel quinoline-chalcogenium compounds. Tetrahed Lett 54: 40-44.

4. Afanas'ev IB, Dorozhko Al, Brodskii AV, Kostyuk VA, Potapovitch Al (1989) Chelating and free radical scavenging mechanisms of inhibitory action of rutin and quercetin in lipid peroxidation. Biochem Pharmacol 38: 1763-1769.

5. Mitchell JH, Gardner PT, McPhail DB, Morrice PC, Collins AR, et al. (1998) Antioxidant efficacy of phytoestrogens in chemical and biological model systems Arch. Biochem Biophys 36: 142-148.

6. Mustapha B, Lhassane I, Isabelle T, Laurence N, Marc P, et al. (2011) Pharmacophore Modelling and Synthesis of Quinoline-3-Carbohydrazide as Antioxidants. Int J med Chem 592879

7. Karali N, Güzel O, Ozsoy N, Ozbey S, Salman A (2010) Synthesis of new spiroindolinones incorporating a benzothiazole moiety as antioxidant agents. Eur J med Chem 45: 1068-1077.

8. Bradshaw TD, Wrigley S, Shi DF, Schultz RJ, Paull KD, et al. (1998) 2-(4-Aminophenyl) benzothiazoles: novel agents with selective profiles of in vitro anti-tumour activity. British Journal of Cancer 77: 745-752.

9. Chua MS, Shi DF, Wrigley S, Bradshaw TD, Hutchinson I, et al. (1999) Synthesis of 2-(4-acylaminophenyl) benzothiazoles and investigations into the role of acetylation in the antitumour activities of the parent amines. J Med Chem 42: 381-392.

10. Bradshaw TD, Stevens MFG, Westwell AD (2001) The discovery of the poten and selective antitumour agent 2-(4-amino-3-methylphenyl) benzothiazole (DF 203) and related compounds. Curr Med Chem 8: 203-210.

11. Cressier D, Prouillac C, Hernandez P, Amourette $C$, Diserbo $M$, et al (2009) Synthesis, antioxidant properties and radioprotective effects of new benzothiazoles and thiadiazoles. Bioorg Med Chem 17: 5275-5284.

12. Kok S, Gambari R, Chui CH, Yuen MC, Lin E, et al. (2008) Synthesis and anti- cancer activity of benzothiazole containing phthalimide on human carcinoma cell lines. Bioorg Med Chem 16: 3626-3631.

13. Rosanna C, Maria L, Calabrò NM, Aaron DS, Moshin A, et al. (2012) Synthesis of benzothiazole derivatives and their biological evaluation as anticancer agents. Med Chem Res 21: 2644-2651.

14. Mohamed AS, Ossama MB, Aliaa MK, Mohamd AA (2008) Synthesis and screening of quinolone antibiotic isosteres. J Chem Res 715-718.

15. Xuan HS, Zhao W, Yong X, Ting HY, Mei D, et al. (2012) Synthesis and Biological Evaluation of Novel Benzothiazole-2-thiol Derivatives as Potentia Anticancer Agents. Molecules 17: 3933-3944.

16. Phoebe FL (2014) Synthesis and antitumor activity of novel pyrazolo[3,4-d] pyrimidine and their tricyclic derivatives. Int J Pharm Sci Rev Res 24(2): 208-214.

17. Manal MK, Sameha MR, Eman KAA, Mohamed AAG, Phoebe FL (2013) Design, synthesis and cytotoxic activity of some novel compounds containing pyrazolo[3,4-d]pyrimidine nucleus. J Chem Sci 125: 1029-1043.

18. Phoebe FL, John NP, Ahmed OE, Lucie R, Jiri G (2015) Design, synthesis and evaluation of novel phthalimide derivatives as in vitro anti-microbial, antioxidant and anti-inflammatory agents. Molecules 20: 16620-16642.

19. Phoebe FL, Waleed AMA, Vaclav B, Lucie R (2016) Novel N-substituted indole Schiff bases as dual inhibitors of cyclooxygenase-2 and 5-lipoxygenase enzymes: Synthesis, biological activities in vitro and docking study. E J Med Chem 123: 803-813.

20. Abdelgawad MA, Abdellatif KRA, Ahmed OM (2014) Design, Synthesis and Anticancer Screening of Novel Pyrazole Derivatives Linking to Benzimidazole Benzoxazole and Benzothiazole. Med Chem S 1: 1-7.

21. Ahmed SA, Ahmed OM, Abdou OA (2014) Synthesis and anti-tumor activities of new $[1,2,4]$ triazolo[1,5-a]pyrimidine derivatives. Eur J Chem 2: 334-338.

22. Vichai V, Kirtikarz K (2006) Sulforhodamine B colorimetric assay for cytotoxicity screening. Nat Protoc 1: 1112-1116.

23. Kumar A, Suchetha KN, Bhargavan D (2012) Evaluation of in vitro antioxidant potential of ethanolic extract from the leaves of Achyranthesaspera. Asian $J$ Pharm Clin Res 5: 146-148.

24. Ahmed SA, Ahmed OM, Elgendy HS (2014) Novel Synthesis of Puriensanalougues and Thieno[2,3-b] pyridine derivatives with anticancer and antioxidant activity. J Pharm Res 8: 1303-1313. 